\title{
Nanotecnología, de la divulgación que tenemos a la participación que queremos*
}

\author{
Paulo Roberto Martins** \\ María Fernanda Marques Fernandes***
}

\begin{abstract}
RESUMEN: El presente texto ofrece una reflexión crítica sobre la experiencia brasileña en la participación pública en nanotecnología. Comienza problematizando la cuestión de la divulgación científica de la nanotecnología para luego dar cuenta de la misión de la Red de Investigación en Nanotecnología, Sociedad y Medio Ambiente (RENANOSOMA) así como de sus estrategias de divulgación y que incluyen: actividades presenciales, producción audiovisual y divulgación vía Internet (chats y web TV), esta última se presenta haciendo énfasis en el programa "Nanotecnologia do Avesso". Después de presentar una evaluación de las estratégias, se reflexiona acerca de la posibilidad de mejorarlas, en particular en lo que se refiere al mencionado programa. Finalmente, se presentan las conclusiones del trabajo.

Palabras Clave: nanotecnología, divulgación científica, participación pública, red de investigación, público no especialista.
\end{abstract}

SUmmaRy: This text offers a critical review on the Brazilian experience in nanotechnology public participation. It begins problematizing the issue of popularizing nanotechnology, describing afterwards the mission of the Research Network in Nanotechnology, Society and Environment (RENANOSOMA) and its outreach strategies which include: classroom activities, audiovisual production, dissemination via Internet (web, chats and TV), with a focus on the program "Nanotecnologia do Avesso." Following an evaluation of the strategies, it then ponders on the possibility of improving them, mainly regarding the webTV program. It finally presents some conclusions.

KEY wORDS: nanotechnology, scientific dissemination, public participation, research network, nonspecialist audience.

\section{Problematizando la divulgación de la nanotecnología}

En la escala de los nanómetros, o la mil millonésima parte de un metro, las sustancias pueden presentar propiedades diferentes a las observadas en el mundo macroscópico. El estudio de esas propiedades y su utilización para producir nuevos materiales y dispositivos constituye las nanociencias y nanotecnologías - o simplemente nanotecnología, término más común para designar esta área de investigación, desarrollo e innovación.

* Traducción por Gian Carlo Delgado.

** Sociólogo, doctor en ciencias sociales, e investigador del Instituto de Pesquisas Tecnológicas del Estado de São Paulo (IPT). Coordinador de la Red Brasileña de Investigación en Nanotecnología, Sociedad y Medio Ambiente (RENANOSOMA) y director y presentador del programa Nanotecnologia do Avesso. Presidente de la ONG Sociólogos Sin Fronteras-América Latina. Correo:<marpaulo@uol.com.br>.

*** Periodista, maestra en ciencias y doctoranda en historia de las ciencias y las tecnologías y la epistemología por la Universidad Federal de Rio de Janeiro (HCTE/UFRJ). Técnica de la Fundación Oswaldo Cruz (Fiocruz).<fernanda.marques@gmail.com>. 
El principio de esta nueva ciencia es que los materiales a escala nanométrica pueden presentar propiedades químicas, físicoquímicas y comportamientos diferentes de aquellos presentados en escalas mayores. Esas propiedades de los nanomateriales están siendo exploradas industrialmente en la fabricación de nuevos cosméticos, medicamentos, pinturas, catalizadores, recubrimientos, tejidos, etc. Sus alcances pueden variar del desarrollo de un simple vidrio a prueba de rayaduras hasta sistemas precisos de entrega y liberación de fármacos. (Rossi-Bergmann, 2008)

Se calcula que existen en el mundo más de mil productos de consumo que contienen nanotecnología. A pesar del numero creciente de productos nanotecnológicos disponibles para el consumo, inclusive en brasil, el asunto es poco conocido por el público general. Una encuesta realizada en Estados Unidos revela que el 37\% de los norteamericanos adultos desconocía la nanotecnología por completo (Project on emerging nanotechnoligies, 2009). Esa desinformación — que debe ser similar para el Brasil- preocupa porque la nanotecnología

tiene consecuencias en todos los aspectos de la sociedad —económicos, éticos, sociales, políticos- así como importantes impactos potenciales en la salud, en el medio ambiente, la alimentación, la cultura y en prácticamente todos las ramas de la industria. (grupo ETC, 2005)

Es también inquietante porque la nanotecnología no es algo aislado. Ella integra la llamada convergencia tecnológica, es decir, la

combinación sinérgica de cuatro grandes áreas de conocimiento: la nanotecnología, la biotecnología, las tecnologías de la información y la comunicación y las ciencias cognitivas (neurociencia), campos que se vienen desarrollando con gran velocidad en las últimas décadas. (Cavalheiro, 2007)

Cada una de esas áreas, aisladamente, tiene el potencial de causar profundos impactos en la sociedad. Combinadas muestran un potencial transformador aún más significativo. Entre las posibles aplicaciones de convergencia tecnológica, Cavalheiro enumera la mejora de la salud y de la capacidad física humana, la mejora de las relaciones sociales y de grupos sociales, la seguridad nacional, la unificación de la ciencia y la educación, la expansión de la cognición y la comunicación humana (Ibid.).

Sin embargo, el autor también llama la atención acerca de la necesidad de estudios sobre los aspectos éticos, legales y sociales de la convergencia tecnológica. En ese sentido, destaca las preocupaciones como las relaciones entre entidades vivas y no vivas y las consecuencias de la manipulación del código genético, entre otras. Para enfrentar esas cuestiones propone una convergencia de las ciencias naturales integradas a las ciencias humanas y a los ciudadanos.

Lo que es cierto, es que la convergencia tecnológica abre posibilidades y encierra dilemas éticos importantes, para que su monopolio pertenezca a cualquiera que sea incluyendo a los científicos. Por lo tanto, nos debe interesar, involucrar y movilizar a todos nosotros. Pues además de ser un tema de interés para la ciencia de punta, ciertamente presupone dimensiones de una nueva ciudadanía planetaria. (Cavalheiro, 2007)

Si hay algo que sea de interés general, precisa entonces, ser ampliamente divulgado. De tal forma, la primera pregunta que se buscará responder es la siguiente: ¿cómo la nanotecnología ha sido divulgada al público en general? 
El prefijo nano, seguido de alguna de otra palabra aparece con frecuencia cada vez mayor en nuestra cotidianeidad. En tanto que esta presencia toma lugar especialmente a través de los medios de comunicación[...] muchas de las noticias parecen prometer que esa presencia será más efectiva en algún futuro más o menos distante, aunque las aplicaciones de nano-esto o de nano-aquello ya están disponibles y mejorando nuestra calidad de vida. (Schulz, 2005)

Es decir: mucha gente ya tiene o tendrá su primer contacto con la nanotecnología a través de los medios de comunicación.

Aunque a veces los medios de comunicación transmiten noticias contrarias a la nanotecnología, la mayoría son optimistas en relación con esta nueva tecnología. Stephens (2005) analizó 350 artículos publicados en periódicos de EUA y de otros países entre 1988 y 2004 ; verificó que $45.95 \%$ de los textos caen en la categoría de "los beneficios y riesgos de la nanociencia y la nanotecnología no discernibles"; $30.92 \%$ se ubican en la categoría de los "beneficios pesan más que los riesgos"; el 10.98\% se ubica en categoría "los riesgos y beneficios precisan ser pensados aunque es cierto que los riesgos son menore y los beneficios son mayores"; $9.94 \%$ en la categoría de "los riesgos pesan más que los beneficios"; $2.60 \%$ en la categoría "límites técnicos para el progreso tecnológico en nanociencia y nanotecnología; ningún límite asociado a las implicaciones éticas, legales y sociales".

Resultados similares fueron obtenidos por Anderson et al. (2005) quienes investigaron el modo en que era enmarcada la nanotecnología en los periódicos. Fueron analizados 344 materiales publicados entre el 01/04/2003 y el 30/06/2004, periodo escogido para el estudio porque comprendió importantes episodios relacionados con la nanotecnología en el Reino Unido, como el inicio de un gran estudio sobre el tema, conducido por la Royal Society y la Royal Academy of Engineering, así como la declaración del Príncipe Charles sobre el tema que desencadenó menciones en la prensa de la plaga gris, misma que se refiere a la noción de que el planeta podría ser destruido por nanomáquinas autorreplicantes fuera de control. A pesar de las referencias a la plaga gris, los resultados muestran que el 38.37\% de los materiales encajaban en la categoría "los beneficios pesan más que los riesgos" y el $11.05 \%$ en la categoría de "los riesgos pesan más que los beneficios".

Aunque los posibles beneficios de la nanotecnología han recibido más atención en los diarios que los posibles riesgos, los materiales estudiados muestran cierta dosis de incertidumbre sobre el tema y las dificultades para abordarlo con un lenguaje adecuado a los lectores y que se podría explicar, en parte, porque los periodistas recurrían a términos e imágenes de ciencia ficción. Aunque la mayoría de los materiales en el periodo investigado haya aparecido en revistas de elite la proporción de textos que encuadran a la nanotecnología en el ámbito de la ciencia ficción y de la cultura popular (16\%) fue idéntica a la de los textos sobre proyectos y descubrimientos científicos. Al respecto de la ciencia ficción fue especialmente prominente en un periodo largo después de la declaración del Príncipe Charles, misma que funcionó de catalizador para que la nanotecnología apareciera en los periódicos durante meses. Ello sugiere cómo una celebridad puede despertar el interés de la prensa por un tema.

Schummer (2005) hizo un análisis de la nanotecnología en los periódicos y en los libros; identificó los libros que el público lee para informarse acerca de la nanotecnología y distinguió entre diferentes tipos de libro y de lector. Entre los libros comprensibles para el público en general, la mayoría no era en investigaciones actuales en nanotecnologíaa, sino más bien sobre previsiones, expectativas y miedos en rela- 
ción con el futuro, así como de oportunidades de negocios. "Debido al número muy pequeño de libros que, de forma competente y comprensible, introduce a los lectores comunes a la investigación corriente y reciente; es esa literatura futurista de varias cualidades la que más que cualquier otra cosa, amolda la percepción pública de la nanotecnología" (Ibid).

En Brasil, el panorama no es muy diferente. Amorim (2008) investigó los materiales publicados en el periódico Folha de Sao Paulo desde la primera vez en que la nanotecnología apareció en el periódico, 1997, y hasta marzo de 2007. Fueron analizados 61 textos: 5 no dejan claro si la nanontecnología traerá beneficios o riesgos; 7 presentaban riesgos potenciales y 49 presentaban beneficios positivos. Los beneficios eran traducidos sobre todo por avances en la medicina y la informática mientras que los riesgos aparecían disfrazados de ciencia ficción, por ejemplo, en referencia a nanorrobots autorreplicantes fuera de control.

Novo et al. (2009) analizaron los discursos sobre nanotecnología en las revistas Epoca y Veja (más específicamente de la nanotecnología aplicada a cosméticos) "según algunos análisis preliminares, percibimos que los reporteros, para hablar sobre el tema, aportan diálogos de científicos y médicos que dan legitimidad a la noticia y que también esos discursos interpelan a los sujetos con el fin de consumir el producto", afirman).

Novo y Borges (2010) encontraron un resultado similar al analizar publicaciones sobre nanotecnología y productos de belleza en la revista Boa Forma. Según las autoras "el discurso de la revista plantea el lado positivo, sin considerar, en ningún momento, que los potenciales consumidores utilicen con cautela el producto, debido al pequeño número de investigaciones en esta área [nanotoxicología]" (Ibid.). Al no existir esta preocupación por parte de la revista en mostrar los dos lados de la noticia, podemos considerar que el marketing es el principal objetivo (Ibid.)

Körbes e Invernizzi (2010) investigaron informaciones sobre nanotencologías presentados en el periódico Fohla de Sao Paulo, en las revistas semanales Veja, Isto É y Epoca, y en las revistas de divulgación científica de Galileu y Super Interessante, de 2002 a 2007, así como en el telediario “Bom Dia Brasil” (TV Globo), en 2009. Las autoras identificaron una serie de promesas asociadas a lo nano, como productos y materiales más eficientes e inteligentes, la promoción de la salud y la calidad de vida; el desarrollo económico y la preservación del medio ambiente. Las autoras llaman también la atención a la reincidencia del determinismo tecnológico, "que promueve una actitud pasiva en relación con los cambios tecnológicos, induciéndonos a pensar en cómo debemos adaptarnos a esos cambios y no cómo debemos direccionarlos" (Ibid).

En otro trabajo, Inverzzi (2008) se enfoca en las visiones de la nanociencia y la nanotecnología diseminadas por el Journal da Ciência e-mail, Boletin de Sociedade Brasileira para o Progresso da Ciência (SBPC). La autora analizó 151 materiales sobre temas vinculados a la NyN entre 2002 y 2007. "Los artículos analizados nos ofrecen algunas visiones del futuro en el que destacan las ideas de revolución, beneficios y creciente eficiencia". La gran mayoría de los artículos tiene como referente a la comunidad científica, en particular los físicos. Solamente 12 textos hacían referencia a organizaciones no gubernamentales, declaraciones del público o de políticos. "Entre las escasas referencias a grupos sociales organizados, encontramos una tendencia a descalificar las capacidades o la legitimidad de las ONGs para opinar sobre nanotecnología", comenta la autora (Ibid.). Por otra parte, apenas 10 investigadores de las áreas sociales y humanísticas fueron citados durante todo el periodo estudiado. 
La visión sobre la nanotecnología encontrada en los medios de comunicación - predominantemente optimista y poco crítica- se refleja en la forma en cómo las personas perciben esa nueva tecnología. Currall et al. (2006) condujeron el primer estudio empírico de larga escala que analizó la percepción del público sobre riesgos y beneficios de la nanotecnología comparando con el caso de otras tecnologías. Después, consultaron por teléfono a 500 personas en EUA constatando que la nanotecnología tiene mayor credibilidad entre el público que los organismos genéticamente modificados, los pesticidas, los desinfectantes químicos, y la ingería genética humana. Por otra, parte una consulta vía web a 4,500 consumidores mostró que, en la evaluación de un producto nanotecnológico, se consideraban los aspectos positivos y negativos conjuntamente. Los participantes se mostraban más preocupados por los riesgos cuando los beneficios del producto no eran tan pronunciados, es decir, ante grandes beneficios, los riesgos preocupan menos.

En Brasil, según una encuesta realizada en 2006 por el Ministerio de Ciência e Tecnología, para el $28 \%$ de los brasileños la ciencia y la tecnología sólo traen beneficios: en 2010 , ese porcentaje subió al 38.9\%. Estos datos se refieren a la ciencia y la tecnología como un todo. Por tanto, se puede esperar un optimismo semejante a la nanotecnología, ello porque

la concepción dominante es que las nuevas tecnologías consiguen generar innovación que a su vez aumentará la competitividad, el crecimiento de la industria y, por lo tanto, del país. Eso va a generar un crecimiento económico, y el crecimiento económico va a generar bienestar. Ésta es la concepción general adoptada hasta ahora por aquellos que impulsan el tema de la nanotecnología. (Martins, 2007)

\section{LA MISIÓN DE RENANOSOMA}

En este contexto y con el objetivo de defender una postura crítica en relación con la nanotecnología, de estimular los debates sobre el tema en el ámbito de las ciencias sociales y promover una participación pública, fue creada, en 2004, la Rede Brasileira de Pesquisa em Nanotecnologia, Sociedade e Meioambiente (RENANOSOMA). La Red está integrada por profesionales de diferentes áreas de conocimiento interesados en analizar otros aspectos de la nanotecnología mas allá de los puramente tecnocientíficos, como lo son sus dimensiones económicas, políticas, sociales, ambientales y éticas.

Esta Red tiene por objetivo hacer que la nanotecnología también sea un objeto de investigación de las ciencias humanas. La nanotecnología viene siendo estudiada mayoritaria y prioritariamente por las ciencias exactas y las ciencias biológicas. Se trata entonces, de hacer que las ciencias humanas se incorporen a esta producción de conocimientos para que podamos tener una visión completa sobre la nanotecnología. Interesa estudiar cuáles son los efectos, los impactos de introducción de esta tecnología en la sociedad y en el medio ambiente. En tanto que la nanotecnología tendrá un impacto bastante importante, es preciso saber de forma anticipada cuáles serán los posibles problemas derivados de la adopción de la nanotecnología. Los temas que entran dentro del ámbito de RENANOSOMA están llegando a pocos, pero gana adeptos: en 2004, la Red contaba con 10 investigadores, de 10 instituciones y para julio de 2011 ya sumaban 34 integrantes de 25 instituciones. La fundación de la Red se dio durante el I Seminario Internacional de Nanotecnología, Sociedad y Medioambiente (SEMINANOSOMA), realizado los días 18 y 19 de octubre de 2004 en la Casa de Cultura 
Japonesa de la Universidad de Sao Paulo. La conclusión del primer SEMINANOSOMA puede ser resumida del siguiente modo:

\begin{abstract}
guiamos nuestros argumentos[...] en torno a la pregunta: ¿qué tanto la sociedad brasileña y mundial están preparadas para la emergencia, en curso, del nuevo paradigma pautado por el desarrollo integrado de las áreas de la nanotecnología, la biotecnología y las tecnologías de la información? Las ricas discusiones de aquel encuentro explicitaron que la respuesta a aquella inquietante pregunta es que ni la sociedad mundial y mucho menos la sociedad brasileña están preparadas para una participación activa y para el direccionamiento de los desarrollos tecnológicos. (Dalcomuni, 2006)
\end{abstract}

Como lo destaca Dalcomuni, las investigaciones en nanotecnología en Brasil están concentradas en áreas de física, química y biología. Sus implicaciones económicas, sociales, legales y éticas, aunque importantes, son desconocidas. Como solución la autora señala la necesidad del desarrollo de la nanotecnología de forma multidisciplinar y con la participación pública en los debates sobre las oportunidades y los riesgos.

\title{
EstrategiAS DE DIVULGACIÓN DE RENANOSOMA
}

Partiendo de la constatación de que los ciudadanos precisan participar en los debates sobre la nanotecnología, la RENANOSOMA viene realizando una serie de acciones para informar y discutir el tema con el público no especialista, asumiendo una visión crítica sobre el asunto. Tales acciones comenzaron a ser desarrolladas de forma más sistemática a partir del llamado (Edital) MCT/CNPq no 12/2006, cuyo objetivo era:

apoyar actividades que propicien la difusión y popularización de la ciencia y la tecnología junto con la sociedad brasileña, y la instalación y fortalecimiento institucional de museos y centros de ciencias y otras iniciativas que tengan como propósito promover la divulgación científica y la mejora de la calidad de la educación informal de las ciencias.

Uno de los proyectos aprobados en ese llamado fue el de Participación Pública en Nanotecnología, propuesto por el coordinador de RENANOSOMA. Incluso después del término de la vigencia de tal llamado, en abril de 2009, el proyecto continúa fructificando gracias a los esfuerzos de los integrantes de la Red. Los resultados pueden ser divididos en 3 categorías: en actividades presenciales, producción audiovisual y divulgación vía Internet.

\section{Actividades presenciales}

Hasta julio de 2011 fueron realizadas cien actividades presenciales en el país y en el extranjero. Muchas fueron desarrolladas gracias a la importante asociación con la Fundación Jorge Duprat Figueiredo (FUNDACENTRO), órgano de investigación del Ministerio de Trabajo y Empleo. Las capitales donde hubo actividades presenciales fueron Porto Alegre, Florianópolis, Curitiba, Sao Paulo, Río de Janeiro, Bello Horizonte, Vitória, Salvador, Aracajo, Recife Joâo Pessoa, Natal, Fortaleza, Belém, Brasilia, Cuiabá, Campogrande y Manaos. También se realizaron actividades en Sâo Leopoldo Itajai, Blumaneao, Cascabel, Marília, Campinhas Piracicaba, Sâo Carlos, Riberirâo Preto e Volta Redonda. 
Las actividades presenciales del proyecto pueden ser comprendidas como momentos de encuentro con el público no especialista para presentar y discutir diversos aspectos de la nanotecnología así como espacios con investigadores de las ciencias exactas y biomédicas, con el objeto de colocar la nanotecnología desde la perspectiva de las ciencias humanas. Entre las actividades realizadas destacan cuatro ciclos de formación en nanotecnología para profesores de nivel medio del estado de Sao Paulo, desarrolladas en conjunto con el Sindicato de Profesores de Educación Oficial del Estado de Sao Paulo (APEOESP).

El primer ciclo de formación se dio en julio de 2008, cuando unos cien profesores se informaron y debatieron sobre nanotecnología en el ámbito de grandes áreas temáticas, como educación, medio ambiente, química, ética, física, sociología y el mundo del trabajo, con la participación de la Universidad de Sao Paulo y uno del IIEP (Intercambio, Informaciones, Estudios e Investigaciones) además del coordinador de RENANOSOMA. Ésta fue sin duda, una de las principales actividades del proyecto de participación pública: por primera vez fue realizado en el país un ciclo de formación en nanotecnología para profesores de nivel medio de la red pública, permitiendo que la información científica sobre nanotecnología llegara a los profesores y, por medio de ellos, a los alumnos.

El segundo ciclo de formación se llevó a cabo en noviembre de 2008 y fue realizado bajo el mismo molde del primero, incluyendo, además, temas como salud y seguridad del trabajador, procesos productivos, mercado de trabajo y formación profesional, agricultura y geografía, ello con la participación de profesionistas de FUNDACENTRO y del Instituto de Economía Agrícola de la Secretaría de Agricultura y Abastecimiento del Estado de Sao Paula, además de la Universidad de Sao Paulo, el IIEP y el coordinador de RENANOSOMA. Más de 45 profesores, inclusive algunos de varias ciudades del interior del estado tuvieron contacto por primera vez con el tema de la nanotecnología, convirtiéndose en diseminadores de información en sus escuelas.

El tercer ciclo, en marzo de 2009, tuvo 63 profesores inscritos. Las actividades de los ciclos de formación fueron realizadas los sábados en periodo integral lo que revela un gran interés de los profesores para recibir información sobre nanotecnología y debatir con los conferencistas. La expectativa, por tanto, es que el tema de la nanotecnología sea llevado a las aulas de los profesores de diferentes disciplinas. Algunos incluso han demostrado la intención de elaborar con sus alumnos proyectos de investigación sobre nanotecnología. Entre las diferencias del tercer ciclo, se puede mencionar el involucramiento de una profesora de la escuela técnica Martin Luther King. Ella se inscribió para participar en el primer ciclo de formación, cuando conoció y se hizo socia del proyecto de participación pública. En el tercer ciclo fue llamada para hacer una presentación sobre física y nanotecnología, además, invitó a RENANOSOMA para realizar una presentación en la mencionada escuela técnica y posteriormente para contribuir en la producción de un DVD sobre nanotecnología.

El cuarto ciclo de formación con cera de 50 profesores inscritos se realizó entre abril y junio 2010, también con encuentros los sábados en periodo integral. Destaca de ese ciclo una actividad específica sobre las técnicas de microscopía electrónica que permiten visualizar objetos en tamaño nanométrico. La investigadora María Cecilia Salvadori del Instituto de Física de la Universidad de Sao Paulo fue la responsable de esa actividad. 


\section{Producción audiovisual}

Fueron producidos dos DVDs con recursos del llamado MCT/CNPq nํㅜ12/2006, titulados "Nanotecnologías y el mundo del trabajo" y "Para entender las nanotecnologías". Están dirigidos, respectivamente, a los trabajadores en general y a los estudiantes de nivel medio. Los DVDs proporcionan información, declaraciones, explicaciones y opiniones de investigadores y de otras personas involucradas en el tema. Los videos están disponibles para su descarga gratuita en el sitio de RENANOSOMA < www.nanotecnologiadoavesso.org>. Se estimula que no sólo sean vistos sino también copiados para darle el mayor alcance posible al público. Centenas de copias ya fueron distribuidas en actividades oficiales del proyecto. La Red tiene también otros dos DVDs gracias a la colaboración con Video Maker Alexandre Quaresma: "Nanotecnología: el futuro es ahora" y "Reflexiones sobre nanotecnología".

\section{Divulgación vía Internet}

En la década de 1990 el Internet dejó de ser una provincia 'habitada' por un pequeño grupo de fanáticos de las computadoras al convertirse en un recurso de consumo de masas para diez millones de estadounidenses. Por otra parte, el Internet evolucionó de simples canales de texto para distribuir datos digitalizados de datos, voz y video. En el proceso se ha presentado un desafío de cómo todo el sector de los medios de comunicación produce y comercializa sus productos[...] la cuestión decisiva es cómo todas estas posibilidades nos pueden beneficiar en una democracia postindustrial. (Dizard, 2000)

De acuerdo con la Comisión Económica para América Latina (CEPAL) es creciente el número de brasileños que usan Internet: en 2001, 4 millones de domicilios (8.5\%) estaban conectados, para el 2007 ese número había aumentado a 11.4 millones (20.1\%). El número de personas en el país con acceso a Internet en cualquier ambiente (domicilio, trabajo, escuelas, café Internet u otros) llegó a 73.9 millones al cierre de 2010, un crecimiento de 9.6\% en relación con el año anterior (IBOPE, 2011). Brasil tiene la mayor población en línea de América Latina y también el que pasa más tiempo navegando por la web: un promedio de 24.3 hs. por internauta en diciembre de 2010 (COMSCORE, 2011).

Sin embargo, entre los países de América Latina y el Caribe, Brasil es también líder en la desigualdad de acceso a Internet: entre los más ricos, el uso es del 52\%, mientras que entre los más pobres es de 1.7\%. (Agência Brasil, 2009). A pesar de esa limitación no se puede ignorar el potencial de Internet para divulgación científica, y en ese sentido el proyecto de proyecto de participación pública aquí relatado, representa contribuciones experimentales para la diseminación de una visión crítica acerca de la nanotecnología, La divulgación vía Internet del proyecto incluye, más allá del propio sitio de RENANOSOMA < www.nanotecnologiadoavesso.org >, chats (ya extintos) y el programa Nanotecnologia do Avesso (en plena actividad).

\section{A) CHAT}

Aunque ahora extinto, el chat representa una experiencia enriquecedora para el proyecto y por eso son aquí comentados. De abril de 2007 a noviembre de 2008, se registraron 164 chats - los archivos correspondientes pueden ser accesados en la página 
del proyecto-. En cada chat un entrevistado - investigador, u otra persona cuyo trabajo estuviese relacionado con la nanotecnología- conversó con los internautas sobre la temática nano, ya sea en términos generales, o sobre un aspecto específico referente a un tema, dígase nanotecnología en alimentos, nanotecnología y los trabajadores, nanotecnología y las ciencias humanas, nanotecnología en cosméticos, nanotecnología y tuberculosis, nanotecnología en los medios, etc. El día, horario y perfil del entrevistado, así como las instrucciones para acceder al chat eran difundidos por medio del "boca en boca", el envío de correos electrónicos y la publicación de noticias. El promedio de los participantes en los chats para todo el periodo fue de 8.3 (aunque hubo asistentes más frecuentes, los participantes no eran siempre los mismos). La participación récord se dio el 17 de octubre de 2007, cuando se invitó a Nilton Morimoto, del Departamento de Ingeniería de Sistemas Electrónicos de la Universidad de Sao Paulo, ahí se registraron 24 personas. Ese récord se logró, sobre todo, gracias a la publicidad que diera un día antes el boletín de la agencia FAPESP.

En marzo de 2008, se realizó una encuesta en la que participaron 30 internautas, con el objetivo de evaluar la estrategia de divulgación en nanotecnología a través de chats. La mayoría pensó que los chats eran válidos, pero preferían otros medios como reportajes y videos. El proyecto de participación pública buscó entonces nuevas alternativas para divulgar lo nano, especialmente la producción de Nanotecnologia do Avesso.

\section{B) WEB TV}

El programa Nanotecnologia do Avesso es una iniciativa de WebTV que se fundamenta en la convergencia de audio, video y datos. La WebTV "abre nuevas posibilidades de interacción social".

Con la misma naturaleza pública abierta y colaborativa de Internet, la Web TV potencia una revolución en la producción, distribución y consumo de información a través de construcciones colectivas en la web, compartiendo con el propio usuario la función de producir y distribuir información, descentralizando la atención de los debates sobre el contenido y la forma y promoviendo un amplio acceso a los flujos informacionales. (Ribeiro, 2009)

El programa se emite los martes de 17:00 a 18:00 hrs por allTV <www.alltv.com. br>, empresa pionera en el género creada en 2002, con una programación muy diversa. Nanotecnologia do Avesso es un programa de entrevistas dirigido al público no especialista con el objetivo de debatir la nanotecnología en sus diversos aspectosbeneficios y riesgos, potencialidades y límites éticos, impactos económicos y al medio ambiente, entre otros. A continuación se presenta un análisis de las 100 primeras ediciones del programa, del 12/01/2009 al 01/02/2011. Se estudiaron los perfiles de los entrevistados y de la audiencia del programa. También se buscó identificar los desafíos para que los pilares de la WebTV — convergencia, flujos de información y construcciones colaborativas - contribuyan para informar, formar y discutir la nanotecnología con el público no especialista desde una perspectiva crítica.

\section{Nanotecnologia do Avesso}

En cada edición de Nanotecnologia do Avesso, el presentador recibe al invitado para discutir aspectos generales o específicos de lo nano. Eventualmente, una misma edi- 
ción reúne a dos o más invitados. Frecuentemente, dos o más ediciones (no sucesivas) cuentan con el mismo invitado. La transmisión es en vivo, desde un estudio o desde donde esté el invitado, mismo que se comunica por medio de Skype. Los internautas pueden enviar sus preguntas o comentarios en "tiempo real" por medio de un chat vinculado al programa. Después de la transmisión los videos quedan disponibles para ser descargados.

Fueron analizadas las 100 primeras ediciones del programa. Sin embargo, debido a problemas técnicos, no fue posible recolectar datos de la audiencia en seis ocasiones, de modo tal que se trabajó con un total de 94 programas.

En promedio, cada edición fue acompañada por 1,089 internautas. Considerándose sólo los programas de 2009, ésa media fue de 1,240 y cayó a 922 a lo largo del año 2010. La figura 1 presenta la audiencia media mensual entre enero de 2009 y enero de 2011. La discontinuidad de la línea se debe a que en marzo de 2010 allTV cambió de servidor, y debido a problemas técnicos, no fue posible registrar el número de internautas. En resumen se observa que la audiencia comienza siendo elevada, sufre una caída y luego vuelve a crecer.

FIGURA 1. Audiência do Nanotecnologia do Avesso

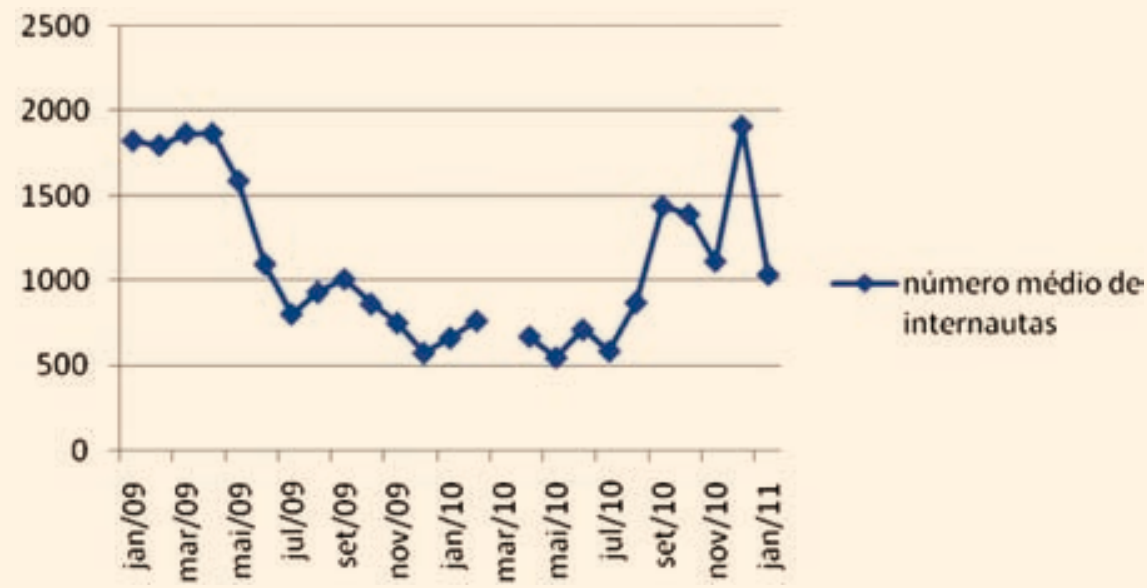

Fuente: Elaboración de los autores.

En relación con los perfiles de los entrevistados, los programas fueron agrupados en diez categorías: "asesorías al movimiento sindical"; "empresa"; "gestor"; "ONG"; "investigador de las ciencias exactas", "investigador de las ciencias humanas"; "investigador de ciencias de la salud"; "representante de los trabajadores", "otros"(por ejemplo, profesora de nivel medio, escritor, etc.); y dos o más (programas que reúnen al mismo tiempo dos o más invitados con perfiles diferentes entre sí). Con base en esa categorización, los "investigadores de ciencias humanas" fueron los invitados más frecuentes (38.3\%), seguidos por los "investigadores de ciencias exactas" (18.1\%) y los "investigadores de ciencias de la salud" (12.8\%). (Véase figura 2). Se trata pues, de una tendencia inversa a la observada en los medios masivos convencionales, donde las ciencias sociales y humanas, en general, no aparecen en temas sobre nanotecnología. 
FIGURA 2. Distribuição dos programas por categorias

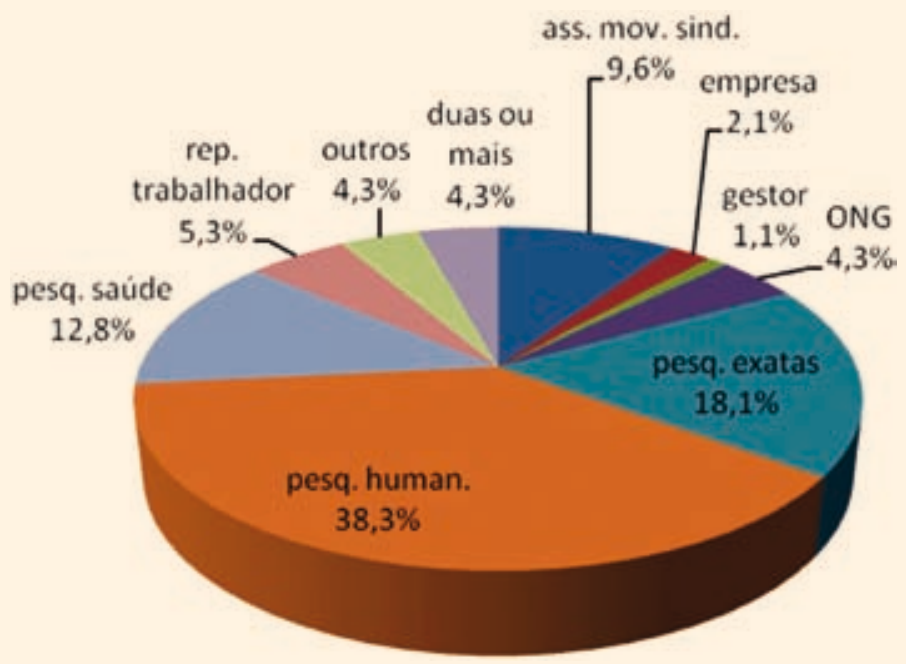

Fuente: Elaboración de los autores.

Aunque en menor proporción, el programa Nanotecnologia do Avesso, también abrió espacio a actores que no son investigadores, tales como los representantes de la categoría "asesoría de movimiento sindical" (9.6\%), "trabajadores" (5.3\%) y "ONG" $(4.3 \%)$ - actores que precisamente casi nunca tienen voz en los medios de comunicación tradicionales.

Al cruzar los datos de la audiencia con los perfiles de los invitados, fue posible calcular la audiencia media por categoría de programa, tal y como se muestra en la figura 3. A grosso modo la audiencia media de todas las categorías se colocó en el rango de los 1,000 y 1,200 internautas. Rango en el que se sitúa el promedio general por programa de todo el periodo analizado $(1,089)$.

Sólo tres categorías escapan a la media anterior: "empresa" y "gestor" por debajo y "asesoría al movimiento sindical", por arriba. Sería prematuro e incorrecto afirmar que las categorías " empresa" y "gestor" tuvieron una tendencia de audiencias menores, en tanto que sólo representan, respectivamente, 2.1 y 1.1\% de las ediciones del programa. No obstante, se puede sugerir que la categoría "asesoría al movimiento sindical”, responsable de caso del 10\% de las ediciones, representó una tendencia a mayores audiencias.

Es interesante analizar esos resultados a la luz de otra investigación (Martins, 2007b) que buscó identificar la comprensión de diferentes segmentos sociales sobre las relaciones entre nanotecnología, sociedad y medio ambiente. Con ese fin se entrevistó, en Sao Paulo, Minas Gerais y el Distrito Federal, a representantes de cinco segmentos: líderes investigadores involucrados en la producción de nanociencia y nanotecnología ("académicos"); liderazgos gerenciales de ciencia y tecnología, medioambiente y gestión estratégica en el ámbito del aparato estatal ("políticas públicas"); liderazgo de empresa de base tecnológica en nanotecnología de asociaciones empresariales ("empresas"); liderazgos de organizaciones no gubernamentales 
FIGURA 3. Audiência média por categoria de programa

\title{
$\mathrm{n}$ \% médio de internautas.
}

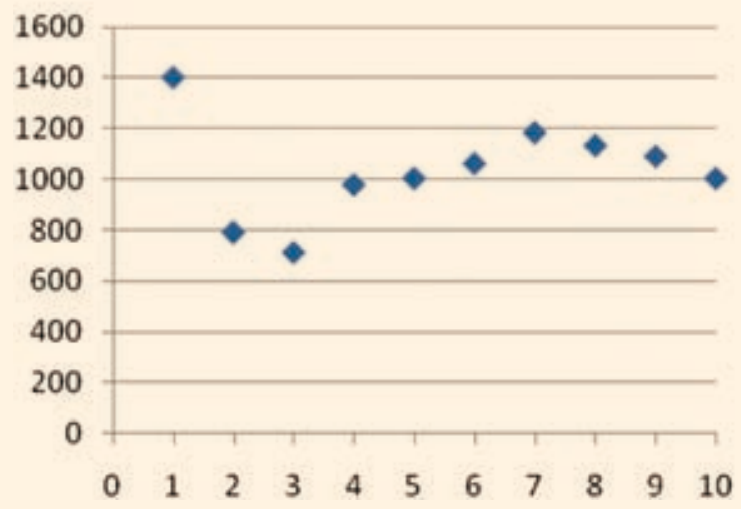

\author{
Categorias: \\ 1-ass. mov, sind. \\ 2-empresa \\ 3-gestor \\ 4-ONG \\ 5-pesq. exatas \\ 6-pesq. human. \\ 7-pesq. saude \\ 8-rep. trabalhador \\ 9-outros \\ 10-duas ou mais
}

Fuente: Elaboración de los autores.

que actúan en defensa de intereses difusos de la sociedad (ONGs); y líderes de sindicatos de trabajadores, centrales sindicales u otras entidades de asesoría (sindicatos). Los encuestados respondieron a preguntas sobre varios temas, incluyendo cuestiones de comunicación.

Por diferentes razones todos los segmentos consideraron importante la divulgación de los conocimientos de nanotecnología para la sociedad. A pesar del discurso a favor de la divulgación aún existen en Brasil importantes dificultades para informar al público no especializados sobre la nanotecnología, sobre todo en relación con los actores del segmento "políticas públicas" (categoría equivalente a "gestores" en este trabajo y que aquí se entienden como gestor público, categoría representada, por ejemplo, por los secretarios, coordinadores y presidentes de órganos ligados a la definición de políticas públicas de ciencia y tecnología). En el caso de Nanotecnologia do Avesso, aunque la producción del programa invitó varias veces a gestores públicos, éstos, por diversos motivos, declinaron la invitación. A lo largo de las primeras cien ediciones, sólo una vez fue posible entrevistar a un gestor y aun así, no era brasileño sino estadounidense.

En relación con la mayor audiencia de los programas con representantes de "asesorías al movimiento sindical" la perspectiva es que, por medio de esas asesorías, los sindicatos, las centrales sindicales y los propios trabajadores se involucren cada vez más en los debates sobre la nanotecnología - algo esencial en la medida en que si un nanocomposite es dañino para la salud, los principales afectados serán aquellos expuestos a las mayores concentraciones del producto por un tiempo más prolongado, esto es, los trabajadores. En ese sentido, cabe destacar con base en la investigación arriba mencionada (Martins, 2007b), que el segmento "sindicatos" demostró estar atento, por un lado, a las posibles polémicas relacionadas con los riesgos en el proceso de trabajo y, por el otro, del papel de la comunicación para minimizar los efectos nocivos de decisiones unilaterales, autoritarias o en beneficio de minorías.

Las ediciones de Nanotecnologia do Avesso fueron también analizadas según el origen del entrevistado. Se constata que en la gran mayoría de los programas los en- 
trevistados eran de Brasil y específicamente de la región sudeste de Sao Paulo (70 de 94 ediciones tuvieron invitados originarios de ese estado). Sólo hubo cinco ediciones con invitados de otras regiones: dos del norte (Amazonas) y tres del sur (Rio Grande do Sul). Incluso de otros estados del sudeste hubo una participación limitada, pues sólo cuatro programas tuvieron invitados de Río de Janeiro y dos de Minas Gerais. Ninguno de Espíritu Santo.

Uno de los desafíos del programa es la diversificación de más voces sobre el tema, incluyendo actores de todas las regiones de los estados del país. Otro desafío importante es aumentar el grado de interacción del programa - en la actualidad se restringe a preguntas y comentarios enviados vía chat-. Una propuesta innovadora de WebTV se basaría en la posibilidad de que los telespectadores/usuarios participen activamente en la producción y emisión de contenido informativo, o sea, que dejan de ser meros receptores de mensajes y pasan a compartir responsabilidades en el proceso comunicacional. Para concretar tal propuesta, Nanotecnologia do Avesso precisa reafirmar su carácter de WebTV, marcado por un "sistema colaborativo vía Internet, creando un ambiente propicio para las construcciones sociales de las más variadas formas de información" (Ribeiro, 2009).

A partir de esta descripción se constató la necesidad de mejorar el programa. Ya se ha dado un primer paso en esa dirección. Se seleccionaron algunos profesionales que ya habían participado en el mismo, en calidad de entrevistados, y se les solicitó que evaluaran diferentes aspectos. Las opiniones y sugerencias - presentadas a continuación- servirán de base para una propuesta de reestructuración, de tal suerte que permita su consolidación como un instrumento de divulgación científica de las nanociencias y las nanotecnologías.

\section{EN BUSCA DE MEJORAS}

En la selección de los profesionales arriba indicados, se buscó seguir dos criterios principales: incluir a entrevistados de perfiles distintos y, siempre que fue posible, de diferentes estados de Brasil. El contacto con los entrevistados se hizo por correo electrónico. Se les solicitó que calificaran los temas y el lenguaje del programa, que identificaran los puntos fuertes y débiles del mismo y que opinaran sobre la televisión por Internet y, finalmente, que proporcionaran sugerencias. Un análisis preliminar de las opiniones y comentarios se resume del siguiente modo:

- A pesar de los diferentes perfiles, que incluyen desde investigadores de las ciencias exactas hasta los representantes del sector sindical, los encuestados consideran que es importante y aprueban que el programa discuta distintos puntos de vista sobre las nanotecnologías, desde los aspectos técnicos hasta los sociales, de las aplicaciones a los riesgos, fomentando así un sentido crítico del público.

- Se reconoce el esfuerzo de la producción del programa en el sentido de que el público no especialista comprenda y participe en el debate, aunque todavía hay obstáculos que deben ser superados, tales como que los expertos aún no consiguen utilizar un lenguaje más sencillo, y la necesidad de emplear otros recursos audiovisuales además de las entrevistas.

- En relación con el soporte (WebTV) se destacaron como ventajas el potencial de interacción con el público y la posibilidad de acceder a ediciones anteriores 
del programa en cualquier momento, así como de poder acceder de forma gratuita.

- Pese a que el uso de Internet en Brasil sea significativo y esté en expansión, los encuestados precisaron que la WebTV no es una práctica tan diseminada en el país, por lo cual, a pesar del alcance mundial de Internet, el programa corre el riesgo de tener audiencia sólo de un grupo selecto de curiosos e interesados, sin poder atender su objetivo de ampliar la difusión del debate sobre las nanotecnologías. Una alternativa para enfrentar este problema sería invertir en la divulgación del programa por medio de asesoría de la prensa, redes sociales, etc.

- A pesar de esta dificultad, los entrevistados mencionaron como punto fuerte del programa, el trabajo de divulgación científica así como el intercambio de experiencias entre los participantes y el establecimiento de una red de contactos.

- Entre las debilidades planteadas, dos merecen especial atención. La primera se refiere al tono, en tanto que a veces se exagera la crítica a la tecnociencia. De acuerdo con lo expresado por los entrevistados, el problema no es la crítica — que debe ser hecha- sino la forma en que esa crítica es planteada. Si ésta es desmedida puede eliminar a los científicos y al público del debate. Para superar ese problema se sugiere aumentar la dinámica de las entrevistas realizando, por ejemplo, en una misma edición del programa un debate entre científicos con visiones opuestas. El segundo comentario se refiere a los internautas que no acompañan el programa semanalmente y que cuando ven una edición aislada se sienten perdidos en relación con el tema de las nanotecnologías. Una opción sería iniciar cada edición siempre con informaciones básicas sobre el tema, proporcionando las bases mínimas para que el internauta se pueda enterar de la discusión. Otra alternativa sería producir diferentes programas de forma segmentada, ello de acuerdo con el perfil del público y teniendo en cuenta la edad y el nivel de conocimiento sobre las nanotecnologías.

- Para hacer más atractivo el programa para el público en general o para proporcionar informaciones básicas, se sugiere destacar el uso de recursos imágenes, gráficos y animaciones.

- Por último, incluso en las informaciones básicas, algunos de los entrevistados hablan de la didáctica de módulos educativos y traducción. De hecho, la comprensión del asunto es fundamental, para que el público no especialistas participe en el debate. Sin embargo, es preciso estar atentos a no caer en una divulgación científica denominada como "modelo de déficit" en la cual los especialistas enseñan a los "legos" una única visión, a menudo con el propósito de valorizar y conseguir el apoyo del público para proyectos de ciencia y tecnología. La propuesta de Nanotecnologia do Avesso se opone a ese tipo de divulgación científica, encaminándose al llamado modelo democrático que "enfatiza el derecho de todos los actores sociales a participar en la toma de decisiones que afectan su vida, incluyendo los aspectos de ciencia y tecnología" y "busca establecer una relación de igualdad entre científicos y el público con el objeto de favorecer la discusión de los asuntos científicos y tecnológicos" (Cavichiolo, 2008).

\section{Consideraciones finales}

Debe destacarse la necesidad de popularizar la nanotecnología de modo que el público no especialista comprenda el tema, desde sus diversos ángulos, y pueda partici- 
par en las discusiones. Consciente de esa necesidad, la RENANOSOMA realiza, desde 2007, el proyecto "Participación Pública en Nanotecnología" que busca disminuir la brecha entre la academia y el público en general.

Las actividades del proyecto, en el ámbito del llamado MCT/CNPq no12/2006, demuestran que la divulgación de lo nano no tiene un costo elevado. Considerándose el número total de participantes de los chats (cerca de mil) los visitantes del sitio RENANOSOMA (cerca de treinta mil hasta abril de 2009) y de internautas conectados al programa Nanotecnologia do Avesso (en torno a los sesenta mil a lo largo del 2009), así como a las centenas de personas que acompañaron las actividades presenciales, se puede decir burdamente que la inversión realizada fue menor a un real por ciudadano (sesenta centavos de dólar), para informarlo sobre el tema. Con más inversión ciertamente se pueden ampliar y mejorar las acciones de divulgación científica de la nanotecnología.

Incluso después de la finalización del proyecto MCT/CNPq nำ12/2006, las actividades del mismo, continuaron, especialmente a través de la realización del programa Nanotecnologia do Avesso. Es de destacarse que esa iniciativa representa un salto cuantitativo de la audiencia que pasó de menos de diez internautas por chat a más de mil por edición de programa de TV vía Internet. Se ha abierto un espacio para una diversidad de voces sobre la nanotecnología y se ha dado también un salto cualitativo en la calidad de la divulgación científica. Después de todo

crear oportunidades para discutir cuestiones éticas de la ciencia, sus riesgos y limitaciones es fundamental para que los jóvenes se conviertan en ciudadanos más conscientes y capaces para tomar decisiones. Además, debemos presentar a los jóvenes una imagen de la ciencia que refleje su complejidad en la que diversos actores, instituciones y muchos recursos financieros están involucrados. (Massarani, 2010)

Pese a todo, y tal y como los colaboradores de Nanotecnología do Avesso han notado, el programa tiene sus limitaciones que habría que atender.

En Brasil, hay otras iniciativas de divulgación de la nanotecnología, con recursos y objetivos diferentes. Entre ellas, quizás la más conocida sea NanoAventura, una exposición interactiva, desarrollada por investigadores de la Universidad Estatal de Campinas (Unicamp) y el Laboratorio Nacional de Luz de Sincrotrón (LNLS), en el cual hay un espacio atractivo en el que se presenta el mundo nanométrico y sus aplicaciones a través de videojuegos.

Otra iniciativa es la del grupo de investigación del Laboratorio Interdisciplinar de Electroquímica y Cerámica (LIEC) de la Universidad Federal de Sao Carlos (UFSCar), que produjo en una computadora animaciones en tercera dimensión para presentar al público no especializado, de manera accesible y atractiva, los conceptos básicos de la nanotecnología y sus aplicaciones. Las animaciones simulan las contribuciones de investigaciones para aumentar, por ejemplo, la vida útil de los altos hornos de la Companhia Siderúrgica Nacional (CSN), la capacidad de memoria de las computadoras, y la resistencia del grafito de los lápices Faber-Castell.

Estaban asociados a imágenes en 3D varios conceptos científicos y de descubrimiento del mundo molecular. De esta forma, hubo una transposición a la realidad virtual de varios desarrollos tecnológicos en términos de una reacción entre moléculas y la superficie y la excitación de una estructura molecular por electrones produciendo luminiscencia. (Fragalle y Longo, 2004) 
Las animaciones producidas fueron usadas en varios reportajes realizados por el departamento de periodismo de EPTV Central, emisora afiliada a la Red Globo de la televisión del estado de Sao Paul, y exhibidas tanto en televisoras locales como nacionales.

Una rápida comparación de esas dos iniciativas con el Proyecto Participación Pública de RENANOSOMA revela, por un lado, que los videojuegos de NanoAventura y las animaciones en 3D de LIEC tienen un aspecto lúdico que, probablemente, atrae más al público en general y facilita la comprensión de los conceptos cientódicos de la nanotecnología y sus aplicaciones. Por otro lado, el proyecto de RENANOSOMA tiene una importante diferencia al aportar un abordaje crítico de la nanotecnología, llamando la atención sobre los posibles riesgos para la salud y el medio ambiente, así como acerca de las implicaciones sociales y éticas de esa nueva tecnología y de la convergencia tecnológica, buscando establecer el diálogo interdisciplinario entre las ciencias naturales y las ciencias humanas, e incorporando nuevas voces a los debates como lo son las de los sindicatos y las de las ONGs.

Cuatro modelos se han utilizado para describir las actividades de comunicación pública de la ciencia (Lewenstein, 2003). El primero es el modelo de déficit que presupone una audiencia que no sabe de ciencia y que precisa llenar este vacío en su educación. Asimismo, aquellos que tienen conocimiento - los científicos- deben "enseñar" a ese público desinformado. Son múltiples las críticas que se han hecho a este modelo y como respuesta se han desarrollado los otros tres modelos: el contextual, el de conocimiento lego y el de participación pública.

En el modelo conceptual —considerado un modelo de déficit más sofisticado-, la audiencia que no sabe de ciencia, para recibir la información científica no sólo simplemente la absorbe sino que además la relabora de acuerdo con sus experiencias previas, su contexto cultural y sus características personales. Este proceso de relaboración explicaría por qué aún en posesión de información científica, un individuo no siempre adopta las actitudes más aterrizadas científicamente.

El modelo de conocimiento lego puede ser visto de dos maneras. Por un lado, sería una adaptación del modelo conceptual: el conocimiento lego sería insertado en la planeación de actividades de acción pública de la ciencia como una estrategia para controlar aquel proceso de relaboración. Por otro lado, se puede asumir que el conocimiento lego es tan relevante como el conocimiento científicotécnico al momento de resolver un problema.

Finalmente, a medida que la opinión pública adquiere peso en las decisiones políticas en ciencia y tecnología, emerge, entonces, un modelo de participación o compromiso público, aquí entendido como el involucramiento de los ciudadanos en la decisión de los rumbos que ha de tomar la ciencia y la tecnología. Aunque este modelo pareciera ser más democrático, también representa un problema: no hay un ciudadano que pueda participar críticamente en ese proceso político si no comprende los contenidos científicos que están en discusión.

Llevando el debate de vuelta al campo de la nanotecnología, ¿cómo los ciudadanos pueden participar cuando muchos no saben lo que significa la nanotecnología ni pueden comprender el concepto de lo que es una molécula? Esa pregunta sugiere que tal vez el mejor camino sea combinar los diferentes modelos de comunicación pública de la ciencia. En el caso de la nanotecnología, se considera que sería bastante interesante 
la articulación de, por ejemplo, los videojuegos de NanoAventura, las animaciones 3D de LIEC y el proyecto de Participación Pública de RENANOSOMA.

\section{BiBLIOgRAFía}

Rossi-Bergmann, Bartira. (2008). "A nanotecnologia: da saúde para além do determinismo tecnológico". Ciência e Cultura, São Paulo, vol. 60, n. 2: 54-57, abril-junio.

Project on Emerging Nanotechnologies. (2009). Nanotechnology, Synthetic Biology, \& Public Opinion: A Report Of Findings, en: <www.nanotechproject.org>. Consultado el $15 / 4 / 2011$.

Grupo etc. 2005. Nanotecnologia: os riscos da tecnologia do futuro. Porto Alegre: L\&PM.

Cavalheiro, Esper A. (2007). "A nova convergência da ciência e da tecnología”. Novos estudos (Cebrap), São Paulo, n. 78, pp. 23-30, Julio.

Schulz, Peter. (2005). "O que é nanociência e para que serve a nanotecnologia?" Física na Escola, v. 6, n. 1: 58-62.

Stephens, Lowndes. (2005). "News narratives about nano S\&T in najor U.S. and non-U.S. newspapers". Science Communication, v. 27, n. 2:175-199.

Anderson, Alison. (2005). "The framing of nanotechnologies in the british newspaper press". Science Communication, v. 27, n. 2: 200-220.

Schummer, Joachim. (2005). "Reading nano: The public interest in nanotechnology as reflected in purchase patterns of books". Public Understanding of Science, v. 14, n. 2: 163-183.

Amorim, Tade-Ane de. (2008). "Nanotecnologia na imprensa: análise de conteúdo do jornal Folha de São Paulo". Em Tese, v. 4, n. 2 (2): 20-36, enero-julio.

Novo, Magda Suzana; Borges, Elizandra Luçardo; Geracitano, Laura Alícia; Henning, Paula Corrêa. (2009). Análise de discurso na mídia: Nanotecnologia, uma nova revolução. III Simpósio Nacional de Tecnologia e Sociedade: Curitiba, Brasil. Disponible en: <www. nanosociedade.com.br Consultado el 15/4/2011>.

Novo, Magda Suzana; Borges, Elizandra Luçardo. (2010). Nanotecnologia e as constituições de gênero. VIII Congresso Iberoamericano de Ciência, Tecnologia e Gênero: Curitiba, Brasil. Disponible en: <www.ppgte.ct.utfpr.edu.br/eventos/cictg> Consultado el 15/4/2011.

Körbes, Clecí; Invernizzi, Noela. (2010). Tecnologia e a difusão da ideia de progresso da ciência. VIII Jornadas Latinoamericanas de Estudios Sociales de la Ciencia y Tecnología: Buenos Aires, Argentina. Disponible en: <www.esocite2010.escyt.org>. Consultado el 15/4/2011.

Invernizzi, Noela. (2008). Visões de futuro: nanociência e nanotecnologia no Jornal da Ciência. VII Jornadas Latinoamericanas de Estudios Sociales de la Ciencia y Tecnología: Rio de Janeiro, Brasil. Disponible en: <www.necso.ufrj.br/esocite2008>. Consultado el 15/4/2011.

Curral, Steven et al. (2006). "What drives public acceptance of nanotechnology?". Nature Nanotechnology, v. 1: 153-155.

Ministério da Ciência e Tecnologia (MCT): <www.mct.gov.br>.

Martins, Paulo Roberto. (2007). “Desenvolvimento recente da nanotecnologia no Brasil: reflexões sobre a política de riscos, impactos sociais, econômicos e ambientais em nanotecnología” en: Emerick, Maria Celeste, Montenegro, Karla B. M. y Degrave, Wim 
(orgs.). Novas tecnologias na genética humana: avanços e impactos para a saúde. Rio de Janeiro, Brasil: Gestec-NIT/Fiocruz.

Dalcomuni, Sônia Maria. (2006). "Inter-relações fundamentais para o desenvolvimento sustentável" en: Martins, Paulo Roberto (org.). Nanotecnologia, sociedade e meio ambiente - Trabalhos apresentados no Segundo Seminário Internacional (II Seminanosoma). São Paulo: Xamã: 49-68.

EDITAL MCT/CNPq no 12/2006 - Seleção Pública de Projetos para Apoio a Projetos de Difusão e Popularização da Ciência e Tecnologia: <www.cnpq.br/editais/ct/2006/ docs/012.pdf>. Consultado el 13/8/2011.

Dizard, Wilson. (2000). A nova mídia: a comunicação de massa na era da informação. Rio de Janeiro: Jorge Zahar, ed.

Comisión Económica para América Latina (CEPAL): <www.cepal.org>.

IBOPE: <www.ibope.com.br>.

Comscore. (2011). The 2010 Digital Year in Review: Latin America. Disponible en: <www. comscore.com>. Consultado el 15/4/2011.

Agência Brasil. (2009). "Brasileiros pobres têm o menor acesso à internet entre 14 países da América Latina e do Caribe”, en: <http://agenciabrasil.ebc.com.br/noticia/200904-07/brasileiros-pobres-tem-menor-acesso-internet-entre-14-paises-da-americalatina-e-do-caribe>. Consultado el 13/8/2011.

Ribeiro, Daniela Costa. (2009). "WebTV: Perspectivas para Construções Sociais Coletivas.” Biblioteca On-line de Ciências da Comunicação. Disponible en: <www.bocc.uff.br>. Consultado el 27/4/2011.

Martins, Paulo Roberto (coord.); Dulley, Richard Domingues; Azevedo, Regina Maria Bueno de; Sanchez-Júnior, Oswaldo. (2007). Nanotecnologia, sociedade e meio ambiente em São Paulo, Minas Gerais e Distrito Federal. São Paulo: Xamã.

Cavichiolo, Cibele Caroline. (2008). Mapeamento e análise das iniciativas de divulgação científica em nanociências e nanotecnologias em jornais e revistas de ampla difusão. Informe presentado a la Coordinadora de la Iniciación Científica e Integración Académica de la Universidad Federal de Paraná como conclusión de las actividades de inicación científica de Edital 2007-2008. Curitiba, Brasil. Disponible en: <www. nanosociedade.com.br/arquivos/File/PUBLICACOES/Mapeamento_e_analise_de_ iniciativas_de_divulgacao_cientifica_nanoc_nanot_CibeleCarolineCavichiolo.doc>. Consultado el 4/7/2011

Massarani, Luisa. (2010). Divulgação científica e mídia: jornalismo científico no Brasil: um panorama geral e desafios. In: Salto para o Futuro/TV Escola. Divulgação científica e educação. MEC. Disponible en: <www.tvbrasil.org.br>. Consultado el 15/4/2011.

NanoAventura: <http://www.mc.unicamp.br/nanoaventura/>.

Fragalle, Edilson Pepino y Longo, Élson. (2004). Divulgação de Ciência Básica na TV: uma experiência em terceira dimensão. Ciência \& Comunicação, vol. 1, n. 1. Disponible en: $<$ www.jornalismocientifico.com.br/revista/01/artigos/artigo3.asp>. Consultado el 13/8/2011.

Lewenstein, Bruce V. (2003). Models of public communication of science and technology. Disponible en: <www.dgdc.unam.mx/Assets/pdfs/sem_feb04.pdf>. Consultado el $13 / 8 / 2011$. 